\title{
Experimental Evaluation of Defects in Ferromagnetic Steel Tube by Electromagnetic Sensor Using Square Wave AC Magnetic Field with DC Bias
}

\author{
Makoto Tohara ${ }^{1}$ and Yuji Gotoh ${ }^{2 *}$ \\ ${ }^{1}$ Department of Technical Development, Toa Non-Destructive Inspection Co., Ltd., \\ 8-46 Shinsone, Kitakyushu 800-0211, Japan \\ ${ }^{2}$ Department of Innovative Engineering, Faculty of Science and Technology, Oita University, \\ 700 Dannoharu, Oita 870-1192, Japan
}

(Received September 15, 2020; accepted November 25, 2020)

Keywords: nondestructive inspection, ferromagnetic steel tube, square wave with DC bias

Regular inspections are required for the heat transfer tubes of multitube heat exchangers in power plants and oil plants. Since the number of these steel tubes is large, high-speed inspection is desired. The use of an AC magnetic field is known to be a high-speed method of inspecting nonmagnetic tubes. On the other hand, since large electromagnetic noise is generated in a ferromagnetic steel tube with nonuniform initial magnetic permeability, it is difficult to inspect a steel tube by an electromagnetic inspection method using a minute AC magnetic field. However, the nonuniformity of the initial permeability in the steel tube is reduced by a minute AC magnetic field with a large static magnetic field. In this research, an electromagnetic inspection method for detecting defects in a steel tube by applying a magnetic field that is a combination of a large static magnetic field and a minute AC magnetic field to the steel tube is investigated. We propose an electromagnetic sensor probe using a square wave AC magnetic field with DC bias that is inserted into the steel tubes for inspecting steel tubes. In addition, it is shown that the detection sensitivity of defects in the steel tube when using the square wave AC magnetic field with DC bias is higher than that when using a sinusoidal AC magnetic field with DC bias. Phenomena that occur during the inspection by this method are investigated in detail by the 3D nonlinear finite element method, taking account of the magnetization properties of the minor loop.

\section{Introduction}

Generally, since the inspection of heat transfer tubes of multitube heat exchangers in power plants and oil plants is a full inspection, high-speed and non-destructive inspection technology is required. Currently, an ultrasonic internal rotation inspection system (IRIS) is used for this inspection. ${ }^{(1)}$ The inspection of ferromagnetic steel tubes using an IRIS enables the detection of defects with high sensitivity. However, an IRIS has the drawback of a low inspection speed

*Corresponding author: e-mail: goto-yuuji@oita-u.ac.jp https://doi.org/10.18494/SAM.2021.3093 
$(100 \mathrm{~mm} / \mathrm{s})$. On the other hand, eddy current testing (ECT) using a minute AC magnetic field is applied to inspect nonmagnetic tubes in power plants and oil plants at a high speed without contact. ${ }^{(2)}$ Since electromagnetic phenomena are used in this inspection method, the inspection speed is high, and the inspection is generally performed at a speed of about 1000 $\mathrm{mm} / \mathrm{s}$. However, when normal ECT is applied to ferromagnetic steel tubes, since the magnetic characteristics inside the tubes are nonuniform, large magnetic noise is detected.

Although the magnetic characteristics inside a ferromagnetic steel tube are nonuniform, the nonuniformity is reduced when a large static DC magnetic field is applied to the tube. Therefore, a method of adding a large DC magnetic field to normal ECT has been proposed. ${ }^{(3,4)}$ However, the inspection equipment for this technique is complicated since two kinds of excitation power supply, DC and AC, are needed. In this research, an electromagnetic inspection method using a simple excitation power supply with a square magnetic field with DC bias is proposed.

Generally, a sinusoidal magnetic field is used as the AC magnetic field in ECT. However, it is considered that the detection sensitivity when using a square wave $\mathrm{AC}$ magnetic field is higher than that when using a sinusoidal AC magnetic field. Therefore, we propose an electromagnetic sensor using a square wave AC magnetic field with DC bias that is inserted into the steel tubes. Phenomena that occur in this proposed inspection method are examined in detail by the 3D nonlinear finite element method (FEM) ${ }^{(5)}$ taking account of the magnetization properties of the minor loop in the ferromagnetic steel tube.

\section{Sensor Model and Experimental Conditions}

Figure 1 shows the proposed electromagnetic sensor probe. This probe is composed of two coils for excitation in series for inducing a square wave AC current with DC bias, a pair of differential search coils, and a magnetic yoke made of permendur, which is an alloy of iron and cobalt. The two coils for excitation and the differential search coil have 220 turns $\times 2$ and 80 turns $\times 2$, respectively. Figure 2 shows the excitation current waveform of the square wave

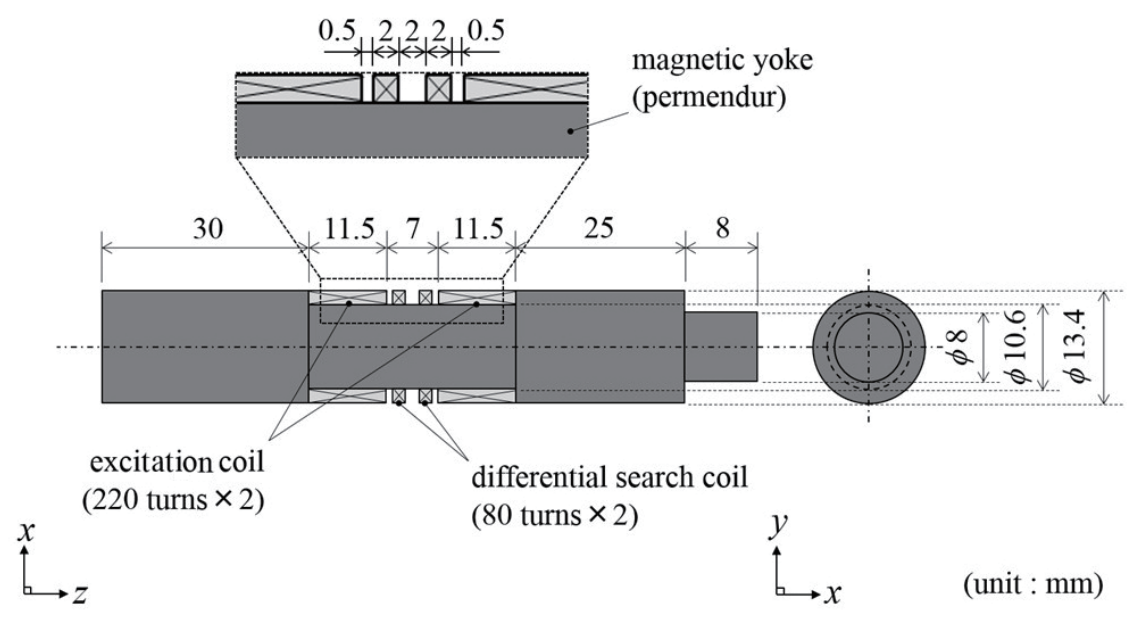

Fig. 1. Proposed electromagnetic sensor. 
current with DC bias. The DC bias, the amplitude of the alternating current of the square waveform, and the excitation frequency are $1.4 \mathrm{~A}, 30 \mathrm{~mA}$, and $100 \mathrm{kHz}$, respectively.

\section{Comparison of Excitation Currents with Sinusoidal Wave and Square Wave}

Generally, a sinusoidal AC magnetic field is applied to generate an excitation current waveform in ECT. In this research, the detection sensitivities of the AC magnetic field of a square wave with DC bias and the AC magnetic field of a sinusoidal wave with DC bias are compared. Figure 3 shows the inspection model for a circumferential defect in the steel tube (STB34SC steel material) using the proposed insertion-type electromagnetic sensor. The outer diameter, length, and thickness of this steel tube with a circumferential defect are $\phi 19 \mathrm{~mm}, 320$ $\mathrm{mm}$, and $2 \mathrm{~mm}$, respectively. The defect width in the $z$-direction and its depth $\left(d_{e}\right)$ are 20 and $0.6 \mathrm{~mm}$, respectively. The distance between the electromagnetic sensor and the inner wall of the steel tube is referred to as the lift-off. In this experiment, the center of the circumferential defect is set as $z=0 \mathrm{~mm}$, and the electromagnetic sensor is moved in the $z$-direction from 30 to -30 $\mathrm{mm}$ in $5 \mathrm{~mm}$ steps, keeping the lift-off at $0.5 \mathrm{~mm}$.

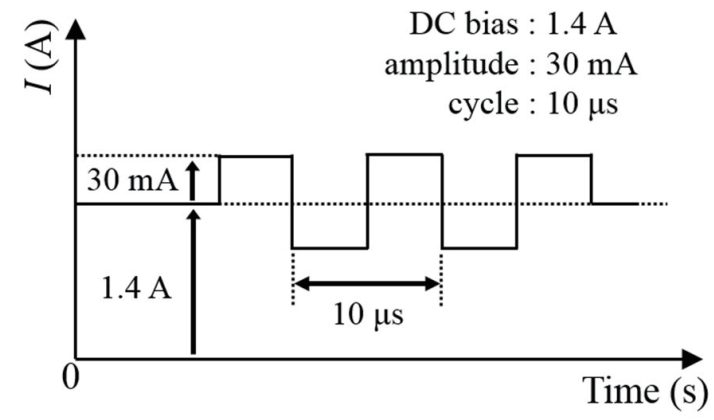

Fig. 2. Excitation current waveform of square wave current with DC bias.

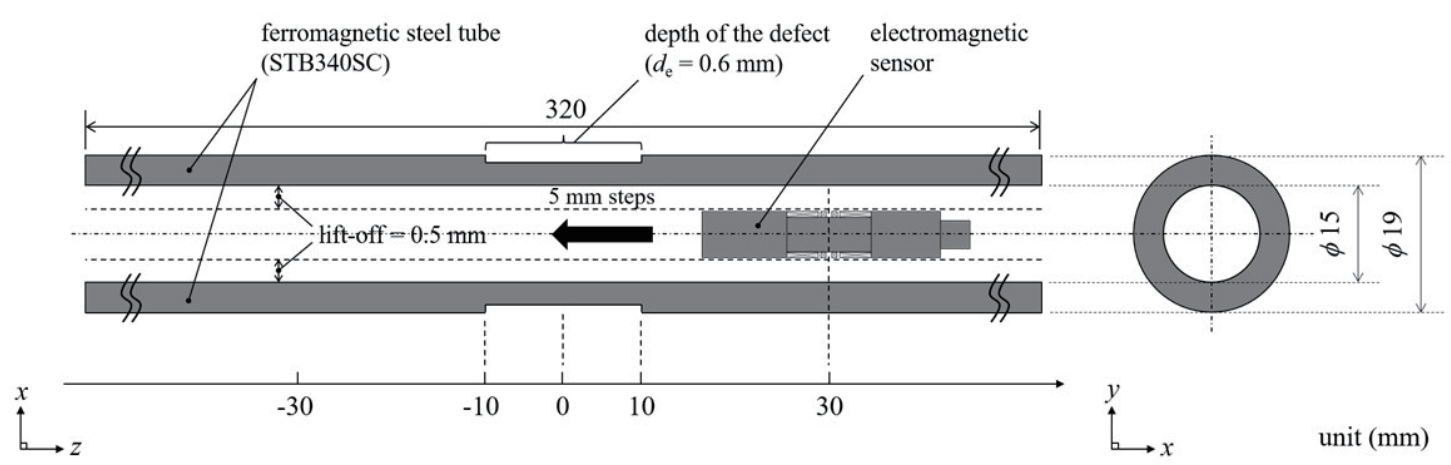

Fig. 3. Inspection model for circumferential defect in the steel tube using the proposed electromagnetic sensor. 
Figure 4 shows the detection sensitivities of the sinusoidal and square wave currents with DC bias. The horizontal axis indicates the position of the electromagnetic sensor and the vertical axis indicates the differential voltage output by the pair of differential search coils in the sensor. This figure shows that the magnitude of the detection signal is increased near both edges of the circumferential defect. In addition, the peak-to-peak value of the detection signal induced by the AC magnetic field is larger for the square waveform than for the sinusoidal waveform. The magnitude of the detection signal for the square waveform is about $27 \%$ larger than that for the sinusoidal waveform. This is because, since the polarity of the magnetic field is changed rapidly when the square wave with DC bias is applied to the steel tube, the magnitude of the eddy current generated in the steel tube is greater than that in the case of the sinusoidal wave magnetic field with DC bias. Moreover, the Fourier series expansion of the square wave, describing the relationship between time and frequency, is given by

$$
\begin{gathered}
f(t)=\frac{4}{\pi} \sum_{n=1}^{\infty} \frac{\sin (2 n-1) 2 \pi f t}{2 n-1}, \\
=\frac{4}{\pi}\left\{\sin (2 \pi f t)+\frac{1}{3} \sin (2 \pi f t)+\frac{1}{5} \sin (2 \pi f t)+\ldots\right\} .
\end{gathered}
$$

These equations show that the square wave can be considered as the sum of a number of sinusoidal wave components whose frequencies are odd multiples of the fundamental frequency. Because the square wave can be considered as the sum of an infinite series of sinusoidal waves multiplied by a coefficient, the magnitude of the detection signal for the square waveform is larger than that for the sinusoidal waveform of the fundamental frequency.

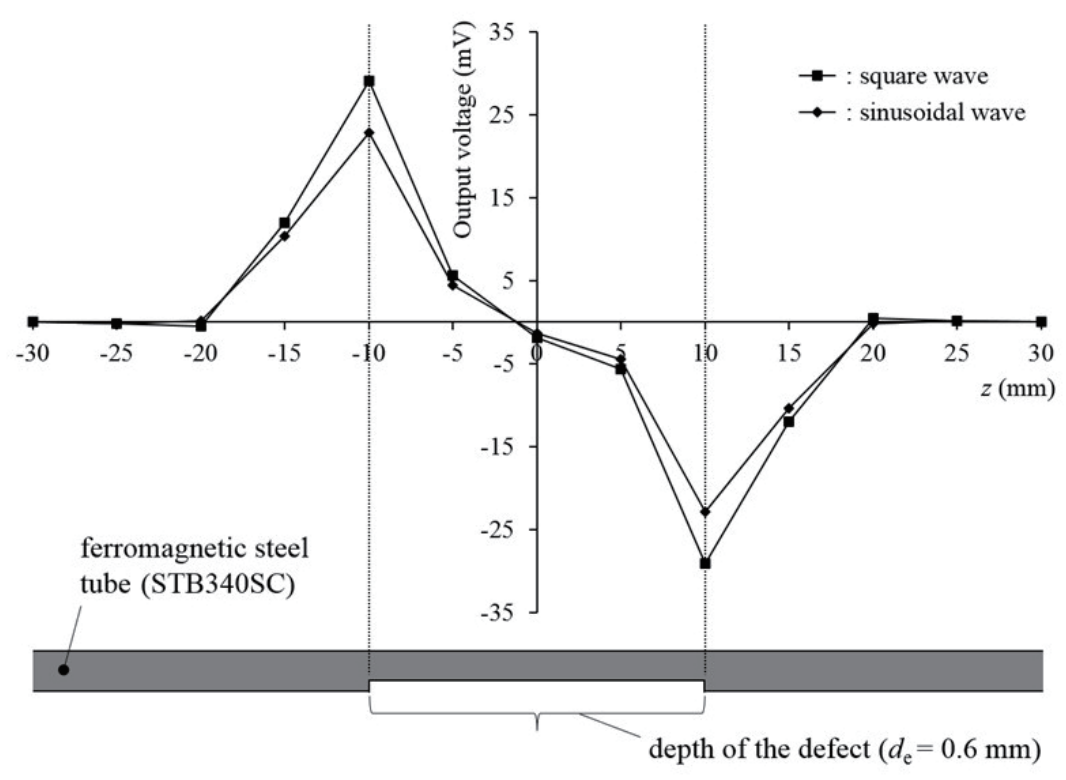

Fig. 4. Detection sensitivities of sinusoidal and square wave currents with DC bias (100 kHz, AC: $30 \mathrm{~mA}, \mathrm{DC}: 1.4 \mathrm{~A})$. 


\section{Experimental Results}

Figure 5 shows the test steel tubes (STB340SC) with inner- and outer-side defects and through holes. The outer diameter and thickness of each steel tube is $\phi 19 \mathrm{~mm}$ and $2 \mathrm{~mm}$, respectively. Figure 5(a) shows a test steel tube with through holes of diameter $\phi 1 \mathrm{~mm}$ to $7 \mathrm{~mm}$. Figure 5(b) shows a test steel tube with inner-side defects at six different depths. The diameter of all these defects is $\phi 3 \mathrm{~mm}$ and the depths of the defects are 10 to $60 \%$ of the thickness of the steel tube. Figure 5(c) shows a test steel tube with outer-side defects at nine different depths. The diameter of all these defects is $\phi 3 \mathrm{~mm}$ and the depths are 10 to $90 \%$ of the thickness of the steel tube. In this experiment, the insertion-type electromagnetic sensor is inserted into the steel tube at a speed of about $1000 \mathrm{~mm} / \mathrm{s}$ while keeping the lift-off at $0.5 \mathrm{~mm}$.

Figure 6 shows the effect of the measured output voltage in the differential search coil when the electromagnetic sensor is moved out of the test steel tube at about $1000 \mathrm{~mm} / \mathrm{s}$. The horizontal axis indicates the moving time of the electromagnetic sensor and the vertical axis indicates the differential voltage output by the pair of differential search coils in the sensor. Figure 6(a) shows the effect of the diameter of the through hole defects in the steel tube in Fig. 5(a). The amplitude of the signal output by the differential search coils increased with the defect diameter. Figure 6(b) shows the effect of the depth of the inner-surface defects in the steel tube in Fig. 5(b). The amplitude of the signal output by the differential search coils increased with the depth of the defect. Figure 6(c) shows the effect of the depth of the outer-side defects on the steel tube in Fig. 5(c). The amplitude of the signal output by the differential search coils increased with the depth of the defect. These figures show that as the defect depth and diameter increased, the amplitude of the signal increased. From these results, it is shown that the inspection speed

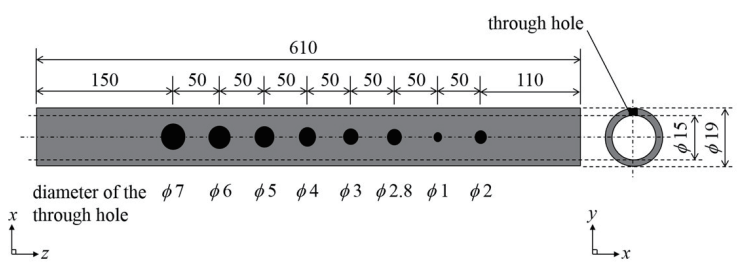

(a)

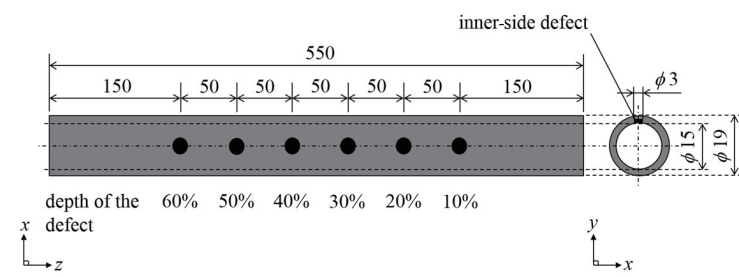

(b)

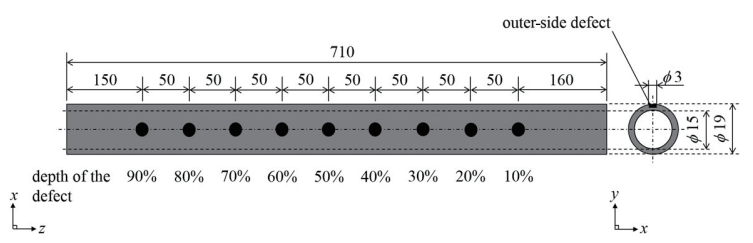

(c)

Fig. 5. Test steel tubes with inner- and outer-surface defects and through holes (STB340SC material). (a) Through hole defect. (b) Defect on the inner side. (c) Defect on the outer side. 


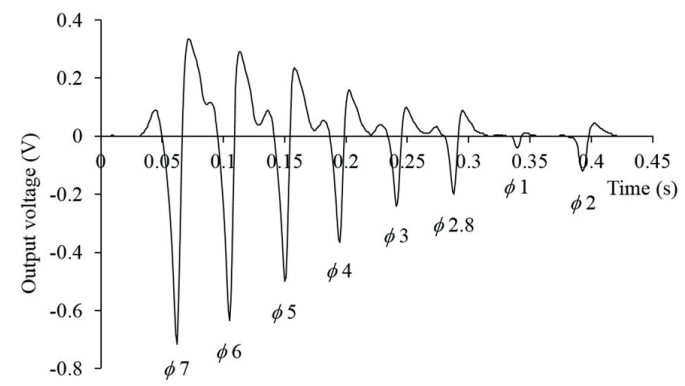

(a)

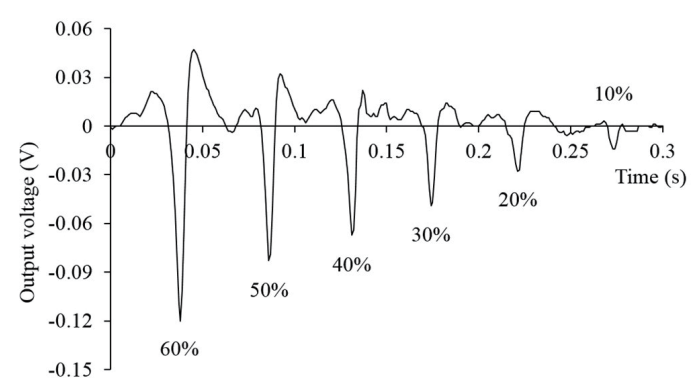

(b)

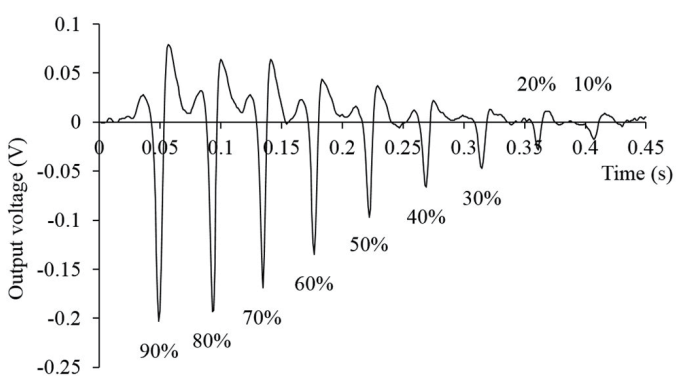

(c)

Fig. 6. Effect of the defect depth or diameter on the measured output voltage. (a) Through hole defect. (b) Defect on the inner side. (c) Defect on the outer side.

of the insertion-type electromagnetic sensor is $1000 \mathrm{~mm} / \mathrm{s}$, which is faster than that in the conventional inspection method applied to the defect inspection of ferromagnetic steel tubes. Furthermore, it is shown that this sensor has a clear signal difference between the part with a defect and the part without a defect. Therefore, this sensor is not easily affected by magnetic noise.

\section{Evaluation by 3D Nonlinear Finite Element Analysis}

\subsection{Conditions of inspection model and analysis}

Figure 7 shows the model of the electromagnetic sensor used for the nonlinear electromagnetic analysis by the 3D FEM. In this inspection method, since a magnetic field using the square wave current with DC bias is applied to the ferromagnetic steel tube, the flux density and eddy current inside the ferromagnetic steel tube are calculated by the 3D nonlinear FEM taking account of the minor loop curve. The play model method ${ }^{(6,7)}$ is used to consider the nonlinear magnetic characteristic of the minor loop in the steel tube. Figure 8 shows the measured scalar hysteresis magnetization curves in the test steel tube made of STB340SC material. These hysteresis curves are used for the 3D nonlinear FEM analysis using the play model method. The hysteresis magnetization characteristics are 80 loops, and the maximum flux density of each loop increases in steps of $0.07 \mathrm{~T}$. Moreover, the initial magnetization curve 


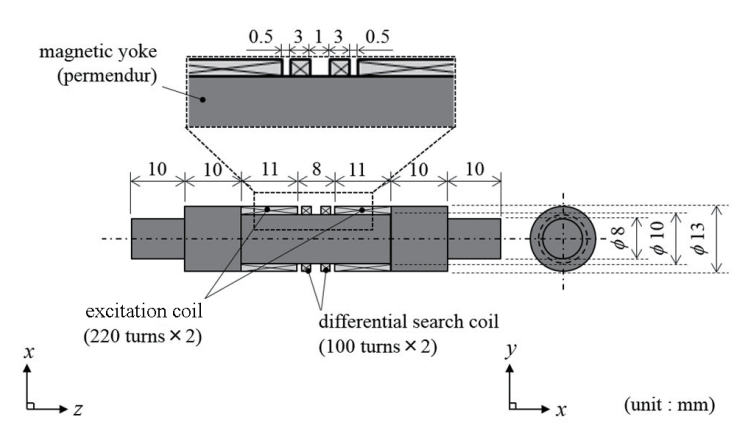

(a)

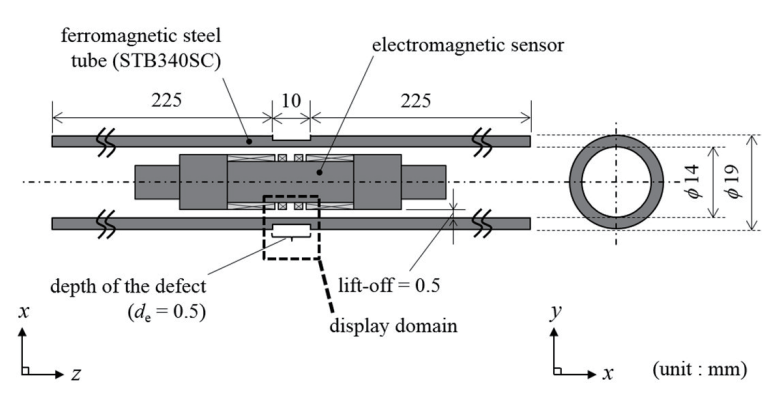

(b)

Fig. 7. Inspection model used for 3D FEM analysis. (a) Electromagnetic sensor. (b) Ferromagnetic steel tube (STB340SC) and outer-side defect.

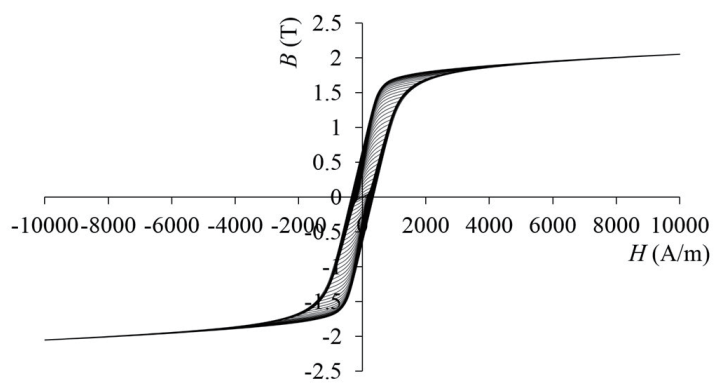

Fig. 8. Hysteresis magnetization curves of steel tube (STB340SC).

of the magnetic yoke of permendur shown in Fig. 9 is used in the nonlinear FEM analysis. In the step-by-step method of the FEM, the time interval $D t$ is chosen as $6.25 \times 10^{-5} \mathrm{~s}$ when the excitation frequency is $500 \mathrm{~Hz}$. In order to obtain the steady-state result, the calculation is carried out over nine cycles (=288 steps). The basic equation used in the electromagnetic field analysis in consideration of the eddy current using the $\boldsymbol{A}-\phi$ method is given by

$$
\begin{gathered}
\operatorname{rot}(v \operatorname{rot} \boldsymbol{A})=\boldsymbol{J}_{0}-\sigma\left(\frac{\partial \boldsymbol{A}}{\partial t}+\operatorname{grad} \phi\right) \\
\operatorname{div}\left\{-\sigma\left(\frac{\partial \boldsymbol{A}}{\partial t}+\operatorname{grad} \phi\right)\right\}=0
\end{gathered}
$$

where $\boldsymbol{A}$ is the magnetic vector potential, $\phi$ is the scalar potential, $v$ is the reluctivity, $\boldsymbol{J}_{0}$ is the current density, and $\sigma$ is the conductivity. The Newton-Raphson (N-R) method is used for the nonlinear iterative calculation of the magnetic characteristic. The calculation and measurement conditions are shown in Table 1 . The ease of calculation is prioritized here to clarify 


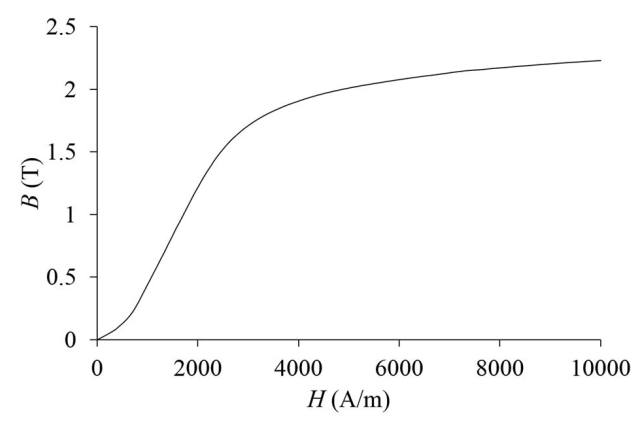

Fig. 9. Initial magnetization curve of magnetic yoke in electromagnetic sensor (permendur).

Table 1

Calculation conditions.

\begin{tabular}{lc}
\hline Excitation coil & 220 turns $\times 2,0.8 \mathrm{~A}(\mathrm{DC}$ bias $), 500 \mathrm{~Hz}, 0.1 \mathrm{~A}_{0-\mathrm{p}}$ \\
\hline $\begin{array}{l}\text { Differential search coil } \\
\text { Lift-off }\end{array}$ & 100 turns $\times 2$ \\
Magnetic yoke & $0.5 \mathrm{~mm}$ \\
Steel tube & permendur, conductivity: $3.33 \times 10^{6} \mathrm{~S} / \mathrm{m}$ \\
& STB340SC, diameter: $19 \mathrm{~mm}$, thickness: $2 \mathrm{~mm}$, length: $460 \mathrm{~mm}$, \\
Outer-side defect & conductivity: $6.66 \times 10^{6} \mathrm{~S} / \mathrm{m}$ \\
Nodes and elements & width $(z$-direction): $10 \mathrm{~mm}$, depth: $0.5 \mathrm{~mm}$, and circumferential defect \\
Convergence criterion & 52585,48704 \\
\hline
\end{tabular}

electromagnetic phenomena occurring in the ferromagnetic steel tube. Therefore, the model size and the frequency and magnitude of the excitation current are different from those in the experiment.

\subsection{Calculation result}

Figure 10 shows only the DC component of the flux density in the square wave magnetic field with DC bias in the steel tube. The display domain of these figures is shown in Fig. 7(b). Figure 10(a) shows the distribution of the flux density inside the steel tube without a defect. The DC flux density component is decreased on the inner surface of the steel tube. Figure 10(b) shows the distribution of the flux density inside the steel tube with an outer-side defect.

For this outer-side defect, the width in the $z$-direction and the depth $d_{e}$ are 10 and $0.5 \mathrm{~mm}$, respectively. Figure 10(b) shows that the DC component of the flux density of the inner surface layer in the steel tube is increased near the defect since the DC component of the flux density is concentrated near the outer-side defect. Figure 10(c) shows the distribution of the DC component of the flux density inside the steel tube with an inner-side defect. For this defect, the width in the $z$-direction and the depth $d_{e}$ are 10 and $0.6 \mathrm{~mm}$, respectively. The DC component of the flux density outside the steel tube near the defect is greater than that without the defect since the DC component of the flux density is concentrated near the inner-side defect. Figure 11 shows only the AC component of the flux density in the square wave magnetic field with DC bias in 


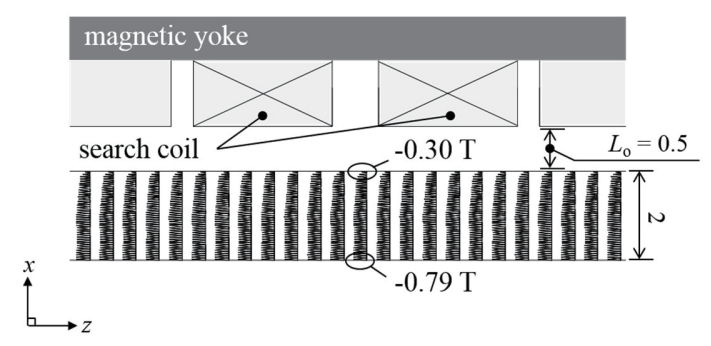

(a)

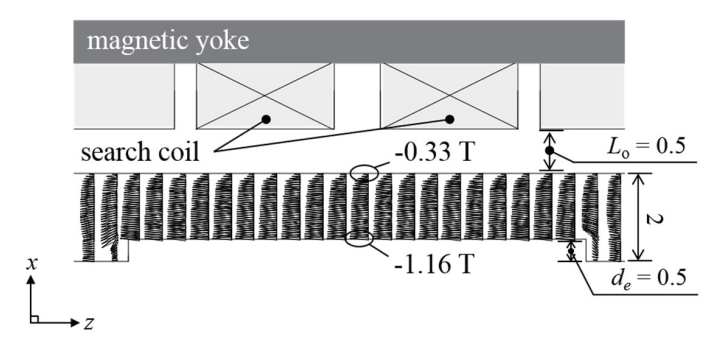

(b)

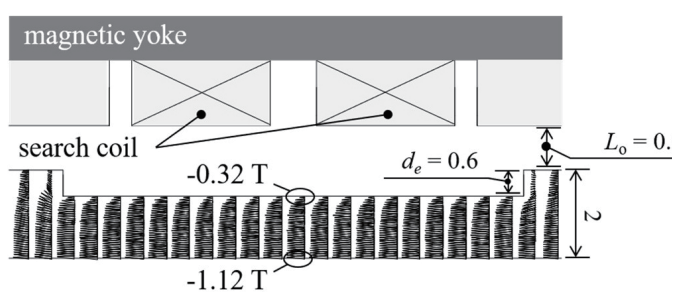

(c)

Fig. 10. Distribution of DC component of flux density in the square wave magnetic field with DC bias in the steel tube. (a) Without the defect. (b) With the outer-side defect. (c) With the inner-side defect.

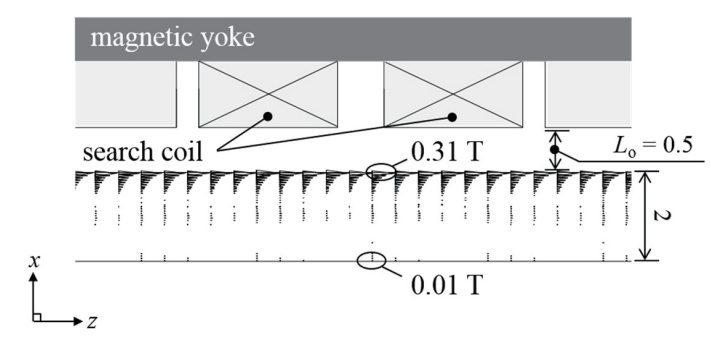

(a)

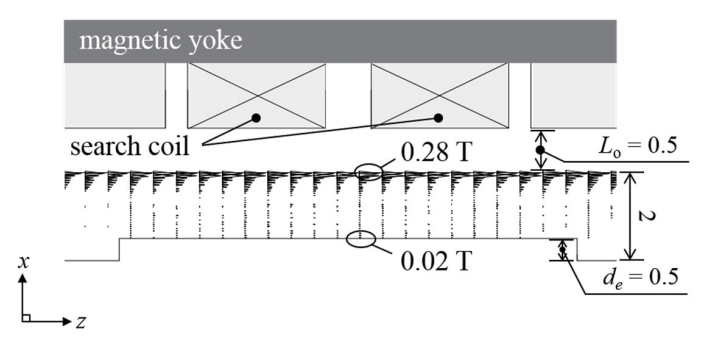

(b)

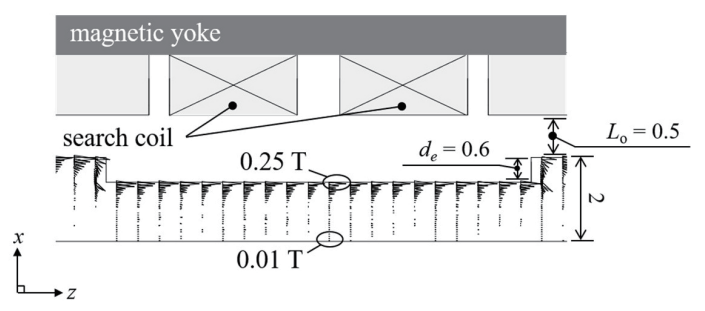

(c)

Fig. 11. Distribution of AC component of flux density in the square wave magnetic field with DC bias in the steel tube. (a) Without the defect. (b) With the outer-side defect. (c) With the inner-side defect.

the steel tube. The display domain of these figures is shown in Fig. 7(b). Figure 11(a) shows the distribution of the flux density inside the steel tube without the defect. The AC flux density component is concentrated on the inner surface of the steel tube by the skin effect. Therefore, as shown in Fig. 11(a), the DC component in the steel tube in the inner-surface domain of the 
steel tube is decreased by the generation of eddy current. Figure 11(b) shows the distribution of only the AC component of the flux density inside the steel tube with the outer-surface defect. The AC component of the flux density in the inner surface of the steel tube is lower than that without the defect. Figure 11(c) shows the distribution of only the AC component of the flux density inside the steel tube with the inner-side defect. The AC component of the flux density in the inner surface of the steel tube is less than that without the defect, similar to the results in Fig. 11(b).

The DC component of the magnetic flux density is concentrated near the outer-side or innerside defects of the steel tube. Since the incremental permeability of the surface layer of the steel tube is reduced, the AC magnetic field component of the square wave on the surface of the steel tube is also reduced. Therefore, the output voltage in the differential search coil in the electromagnetic sensor is changed near the defect in the steel tube.

\section{Conclusions}

The results obtained in this study are summarized as follows.

(1) The inspection speed when using the proposed insertion-type electromagnetic sensor was experimentally shown to be faster than that of the conventional inspection method. In addition, this method was shown to be robust to the magnetic noise of the ferromagnetic material.

(2) When the proposed insertion-type electromagnetic sensor using a square wave magnetic field with DC bias is applied to a ferromagnetic steel tube, it is possible to detect innerside and outer-side defects in the tube. The proposed method is also capable of detecting inner-side and outer-side defects with depths of more than $10 \%$ of the thickness of the tube. However, using this inspection technique, it is not possible to distinguish innerside and outer-side defects in the steel tube. A method of identifying the inner-side and outer-side defects in a steel tube is a future research subject. In addition, it is necessary to experimentally investigate whether the proposed electromagnetic sensor can be applied to defects of various shapes.

(3) Phenomena that occur in the inspection method were clarified in detail by the 3D nonlinear FEM taking account of the magnetizing properties of minor loops. When the square wave magnetic field with DC bias is applied to the steel tube with an inner-side or outer-side defect, the DC component of the flux density on the surface of the steel tube is increased. Therefore, the incremental magnetic permeability of the tube surface is reduced. This is because the DC component of the flux density is concentrated near the defect and is increased at the surface of the steel tube. Then, the AC flux density component of the square wave magnetic field on the inner surface of the steel tube with the defect becomes less than that in the tube without the defect. From the above phenomena, we found that the detection of inner-side and outer-side defects on a steel tube is possible by using the square wave magnetic field with DC bias.

(4) Since the polarity of the magnetic field is changed rapidly when the square wave with DC bias is applied to the steel tube, the magnitude of the generated eddy current in the steel 
tube is greater than that in the case with the sinusoidal wave magnetic field with DC bias. Therefore, the sensitivity of defect detection with the square waveform is higher than that with the sinusoidal waveform of the fundamental frequency.

\section{References}

1 A. Joseph, G. K. Sharma, and T. Jayakumar: J. Pure Appl. Ultrason. 31 (2009) 24.

2 J. Garcia-Martin, J. Gomez-Gil, and E. Vazquez-Sanchez: Sensors 11 (2011) 2525.

3 Y. J. Kim, J.-Y. Yi, and S. Lee: J. Appl. Phys. 60 (1986) 3327.

4 Y. Gotoh, K. Sakurai, and N. Takahashi: IEEE Trans. Magn. 45 (2009) 4467.

5 Y. Gotoh, K. Sakurai, N. Takaoka, Y. Misaka, K. Kawasaki, and N. Takahashi: IEEE Trans. Magn. 47 (2011) 3546.

6 T. Matsuo, Y. Osaka and M. Shimasaki: IEEE Trans. Magn. 36 (2000) 1172.

7 T. Matsuo, D. Shimode, Y. Terada, and M. Shimasaki: IEEE Trans. Magn. 39 (2003) 1361.

\section{About the Authors}

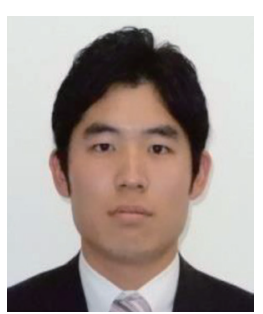

Makoto Tohara graduated from Kyushu Polytechnic College, Japan, in 2016. He received his M.E. degree from Oita University, Japan, in March 2018. Since April 2018, he has been working at Toa Non-Destructive Inspection Co., Ltd., and conducting research as part of his doctoral course at Oita University. His main research interest is nondestructive inspection using electromagnetic phenomena. (v18f1001@oita-u.ac.jp)

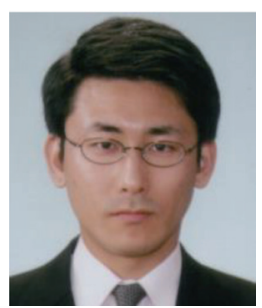

Yuji Gotoh received his B.E. and M.E. degrees from Polytechnic University, Japan, in 1996 and 1998, respectively, and his Ph.D. degree from Okayama University, Japan, in 2002. He is presently a professor in the Faculty of Science and Technology, Oita University. His main research interest is the development of nondestructive electromagnetic inspection methods for steel materials. 